sammenzufassen, die neben der Gruppe der Polyederkrankheiten und der Gruppe der Viruskrankheiten ohne Einschlußbildung (z. B. Sackbrut der Bienen) steht. Die eigenen Ergebnisse scheinen die Ansicht von Steinhaus zu bestärken; künftige eigene vergleichende Untersuchungen mögen zeigen, ob es eine Gruppe von Kapselviren gibt.

Wenn man von der geringen Größe der Viruskapseln absieht, könnte man sie auch mit den Sporen von Mikrosporidien vergleichen, die sehr ähnliche Krankheitssymptome bei Insektenlarven bewirken: die Raupen schwellen an, und beim Anstechen tritt die veränderte, milchig-trübe Lymphe aus, die große Mengen von Sporen enthält. Die Mikrosporidien sind typische Insektenparasiten und ihre Sporen sind stark lichtbrechende Gebilde, die in der Gruppe der Coccosporidien nur etwa $1,2 \mu$ groß sind. Nach $\mathrm{Z}$ w ö lf e $\mathrm{r}^{6}$ schlüpft der Sporoblast bei den Mikrosporidien - ebenso wie die Virusstäbchen (Abb.9) - bei alkalischer Reaktion aus der Spore und wächst im

${ }^{6} \mathrm{~K} . \mathrm{Z}$ w ölf e r, Verh. dtsch. Ges. angew. Entomol. 1926; zit. nach K. Es c h e r i c h, Die Forstinsekten Mitteleuropas, 1931, Bd. III, S. 75.
Zellplasma zu blasigen Gebilden heran. In diesen Blasen bilden sich viele Kerne, aus denen wieder sehr viele dickwandige Sporen entstehen. Es darf aber nicht verkannt werden, daß bei aller Ähnlichkeit, die im Krankheitsverlauf und in der Bildung von Einschlußkörperchen im Plasma besteht, der Unterschied von hochdifferenzierten Sporen und den Viruskapseln zunächst unüberbrückbar erscheint. Die Kapsel- und Polyedervirusstäbchen selbst könnten vielleicht mit Chromosomen verglichen werden, wobei die in den Abb. 9 und 10 auf den Originalbildern noch besser hervortretenden Strukturen an die vielfach beschriebenen Spiralstrukturen erinnern. Allgemein betrachtet, weisen die stäbchenförmigen Kapsel- und Polyederviren durch die Hüllbildung (Elementarkörperchen) und die bündelförmige Ordnung eine höhere Organisationsstufe auf als die molekularen Pflanzenviren vom Typ des ebenfalls stäbchenförmigen Tabakmosaikvirus.

Für anregende Diskussion danke ich Hrn. Prof. Dr. A. K ühn und Hrn. Dr. H. Friedrich-Freksa, Frl. M. Vialon und Frau U. John danke ich für ihre große Hilfe.

\title{
Untersuchungen zur Kollidonwirkung bei Viren (Bergold)
}

\author{
Von Walter Kikuth, Marianne Bock und Rudolf Gönnert \\ Aus dem Chemotherapeutischen Laboratorium der Farbenfabriken Bayer, Werk Elberfeld \\ (Z. Naturforschg. 3 b, 342- 45 [1948]; eingegangen am 25. Dezember 1948)
}

Bei verschiedenen tierischen Virusinfektionen wurde eine in-vitro-Wirkung von Kollidon festgestellt. Diese äußerte sich in allen Versuchen in einer Virulenzabschwächung. Es standen folgende Virusinfektionen der Maus zur Verfügung: murines Fleckfieber, Bronchopneumonie, Lymphogranuloma inguinale, Ektromelie, lymphocytäre Choriomeningitis, Louping ill. Chemotherapeutisch erwies sich Kollidon als wirkungslos. Damit konnten die Ergebnisse von B e r g o l d über in-vitro-Wirkung des Kollidons auf das Polyedervirus von Porthetria dispar bei anderen tierischen Virusinfektionen bestätigt werden.

$\mathrm{K}$ ürzlich berichtete B e r g o l d ${ }^{1}$ über Virulenżabschwächung des Polyedervirus des Schwammspinners, Porthetria dispar. Dieser Virulenzverlust um etwa 2 Zehnerpotenzen trat ein, wenn Polyedervirus in vitro mit Kollidon versetzt wurde. Der Effekt blieb dagegen aus, wenn die Schwammspinnerraupen kurze Zeit vor oder nach der Infektion mit Kollidon behandelt wurden. Beim Kollidon handelt es sich um ein Polymerisationspro-

${ }^{1}$ G. B e r g o l d, Z. Naturforschg. 3 b, 300 [1948]. dukt des Polyvinylpyrrolidons ${ }^{2}$. Auf Anregung von Hrn. Bergold prüften wir bei verschiedenen tierischen Virusarten die in-vitro-Wirkung des Kollidons, um festzustellen, ob es sich bei der Virulenzabschwächung des SchwammspinnerPolyedervirus um eine für Virusarten allgemeine Gesetzmäßigkeit oder um eine spezifische Wirkung auf dieses Insektenvirus handelt.

${ }^{2}$ G. H e c h t u. H. W e e s e, Münch. med. Wschr. 1943, 11. 
Zu diesem Zweck wählten wir Vertreter verschiedener morphologisch-systematisch abgrenzbarer Virusgruppen aus. Wir prüften den Kollidoneinfluß auf den den Bakterien nahestehenden Erreger des murinen Fleckfiebers, Rickettsia mooseri, auf die zu den bläschenförmigen Virusarten (Cysticeten $^{3}$ ) gehörenden Erreger des Lymphogranuloma inguinale und der Bronchopneumonie der Maus, den zu den quaderförmigen Virusarten gehörenden Erreger der Ektromelia infectiosa der Maus, das Influenzavirus, das morphologisch noch nicht bekannte Virus der lymphocytären Choriomeningitis und schließlich auf eine der kleinsten tierischen Virusarten, den Erreger des Louping ill der Schafe. In allen Fällen ließen wir eine 10-proz. Kollidon-Lösung vor der Infektion $1 / 2$ Stde. bei Zimmertemperatur auf die zentrifugierte OrganVirus-Suspension einwirken. Sämtliche Virusarten wurden auf weiße Mäuse, allerdings verschiedener Herkunft, verimpft. Der Infektionsmodus und die angewandte Verdünnung der Virussuspension sind aus Tab. $1 \mathrm{zu}$ ersehen. Neben den in-vitroVersuchen wurde weiter die chemotherapeutische Wirksamkeit des Kollidons bei allen Virusinfektionen, mit Ausnahme der Ektromelie, geprüft. Alle Mäuse wurden hierbei 5 -mal mit $10 \mathrm{mg} / \mathrm{kg}$ subcutan behandelt.

Die Versuchsergebnisse (Kollidon in vitro, Kontrollen, chemotherapeutischer Versuch mit Kollidon) sind in Tab. 1 zusammengestellt. Die in Spalte $V\left(V_{v}, V_{k}\right.$ und $\left.V_{c}\right)$ angegebenen Zahlen geben das Verhältnis von überlebenden Mäusen (Zähler) zu den infizierten Tieren (Nenner) an. Es wurden nur die Mäuse berücksichtigt, die an der Infektion und nicht infolge des Infektionstraumas starben. Je nach Test wurden daher die am 1.-4. Tag nach Infektion gestorbenen Mäuse eliminiert. In der Spalte $M\left(M_{v}, M_{k}\right.$ und $\left.M_{c}\right)$ ist der reziproke Wert des mittleren Todestages (Überlebenszeit) angegeben $\left(M=\frac{\Sigma 1 / t}{n}, t=\right.$ Überlebenszeit in Tagen nach Infektion, $n=$ Zahl der Tiere). Der reziproke Wert wurde gewählt, da bei dessen Berechnung auch die überlebenden Tiere (Überlebenszeit $=\infty, 1 / \infty=0$ ) berücksichtigt werden können. Der reziproke Wert des mittleren Todestages muß um so kleiner gegenüber den Kontrollen sein, je besser die Kollidonwirkung ist. Schließlich wurde noch der Quotient $Q_{v}=\frac{M_{v}}{M_{k}}$ bzw. $Q_{c}=\frac{M_{c}}{M_{k}}$ berechnet (s. Tab. 1). $Q$ muß bei Gleichheit des Verlaufes zwischen Versuch und Kontrollen gleich 1 sein. Der Quotient ist um so kleiner, je ausgesprochener die Kollidonwirkung ist. Wird $Q$ beträchtlich größer als 1 , so kann daraus auf eine ungünstige Wirkung der geprüften Substanz auf den Infektionsverlauf geschlossen werden.

Das Ergebnis der von uns an den genannten Virusarten ausgetesteten Wirkung des Kollidons auf die Virulenz entspricht dem von Bergold ${ }^{1}$ beim Schwammspinnervirus erhobenen Befund der Virulenzabschwächung. Allerdings ist bei der Anordnung unserer Versuche der Grad der Virulenzabschwächung zahlenmäßig nicht exakt erfaßbar. Dies ist einmal darauf zurückzuführen, daß das verwendete Tiermaterial klein ist und daß zum anderen keine Bestimmung der Dosis infectiosa minima vorgenommen werden konnte. Die allgemeine Gesetzmäßigkeit der Virulenzabschwächung bei allen geprüften Virusarten durch in-vitro-Einwirkung von Kollidon kann aber als gesichert angesprochen werden (s. Tab.1). Sie tritt besonders deutlich hervor, wenn man den in-vitro-Versuch mit dem chemotherapeutischen Versuch vergleicht. $Q_{v}$ beträgt im Mittel $0,47, Q$ dagegen 1,39. Bei den meisten in-vitro-Prüfungen ist der Prozentsatz der überlebenden Mäuse gegenüber den Kontrollen erhöht, die mittlere Überlebenszeit verlängert und der Quotient $Q_{v}<1$. Wieweit es sich bei den geringfügigen Unterschieden zwischen den einzelnen Virusarten um verschiedene Grade der Beeinflussung handelt oder nur um Zufallsbefunde, ist auf Grund des kleinen Tiermaterials und der Uneinheitlichkeit im Ausfall der Versuche (s. Vers. 1 und 2 der einzelnen Teste in Tab.1) nicht zu entscheiden. Wir möchten vorläufig das letztere annehmen. Im Gegensatz zu der Virulenzabschwächung im in-vitroVersuch zeigte der chemotherapeutische Versuch keine günstige Beeinflussung des Infektionsverlaufes, was sich auch aus dem um 1 schwankenden Wert für $Q_{c}$ schließen läßt.

Bergold ${ }^{\mathbf{1}}$ hat in seiner Veröffentlichung schon auf die besonderen Eigenschaften des Kollidons hingewiesen, welche sich nach den Untersuchungen von $\mathrm{Schubert}$, und Bennhold ${ }^{4}$ sowie $\mathrm{Schuber} \mathrm{t}^{5}$ über die Wechselbeziehungen

${ }^{3}$ H. R u s k a u. K. P o p p e, Z. Naturforschg. 2b, 35 [1947].

${ }_{4}$ R. S chubert u. H. B ennhold, Z. ges. exp. Med. 113, 722 [1944].

${ }^{5}$ R. S c h u be r t, Klin. Wschr. 26, 143 [1948]. 


\begin{tabular}{|c|c|c|c|c|c|c|c|c|c|c|c|c|}
\hline \multirow{2}{*}{ Test } & \multirow{2}{*}{$\begin{array}{c}\text { Ver- } \\
\text { suchs- } \\
\text { Nr. }\end{array}$} & \multirow{2}{*}{$\begin{array}{c}\text { Infek- } \\
\text { tions- } \\
\text { art }\end{array}$} & \multirow{2}{*}{$\begin{array}{c}\text { Virussusp. } \\
\text { Ver- } \\
\text { dünnung } \\
1:\end{array}$} & \multirow{2}{*}{$\begin{array}{l}\text { Beendigg. } \\
\text { des Ver- } \\
\text { suchs am } \\
\text { Tag n.Inf. }\end{array}$} & \multicolumn{3}{|c|}{$\begin{array}{c}\text { Kollidon } \\
=\text { in-vitro-Versuch }\end{array}$} & \multicolumn{2}{|c|}{ Kontrollen } & \multicolumn{3}{|c|}{$\begin{array}{l}\text { Kollidon = chemo- } \\
\text { therapeut. Versuch }\end{array}$} \\
\hline & & & & & $V_{v}$ & $M_{v}$ & $Q_{v}=\frac{\boldsymbol{M}_{v}}{\boldsymbol{M}_{k}}$ & $V_{k}$ & $M_{k}$ & $V_{c}$ & $M_{c}$ & $Q_{c}=\frac{\boldsymbol{M}_{c}}{\boldsymbol{M}_{k}}$ \\
\hline \multirow[t]{2}{*}{$\begin{array}{l}\text { Rickettsia } \\
\text { mooseri }\end{array}$} & 1 & $\begin{array}{l}\text { i. } p . \\
\text { i. p. } \\
\text { i. p. }\end{array}$ & $\begin{array}{r}50 \\
100 \\
500\end{array}$ & $\begin{array}{l}14 . \\
14 . \\
14 .\end{array}$ & $\begin{array}{r}7 / 12 \\
10 / 12 \\
11 / 12\end{array}$ & $\begin{array}{l}0,044 \\
0,013 \\
0,006\end{array}$ & $\begin{array}{l}0,52 \\
0,26 \\
0,11\end{array}$ & $\begin{array}{l}4 / 12 \\
7 / 12 \\
6 / 12\end{array}$ & & $\begin{array}{l}2 / 12 \\
1 / 12 \\
7 / 12\end{array}$ & $\begin{array}{l}0,115 \\
0,120 \\
0,048\end{array}$ & $\begin{array}{l}1,35 \\
2,40 \\
0,91\end{array}$ \\
\hline & 2 & $\begin{array}{l}\text { i. } p . \\
\text { i. p. } \\
\text { i. p. }\end{array}$ & $\begin{array}{r}50 \\
100 \\
500\end{array}$ & $\begin{array}{l}14 . \\
14 . \\
14 .\end{array}$ & $\begin{array}{l}19 / 24 \\
24 / 24 \\
22 / 24\end{array}$ & $\begin{array}{l}0,017 \\
0,0 \\
0,008\end{array}$ & & $\begin{array}{l}12 / 24 \\
15 / 24 \\
15 / 24\end{array}$ & $\begin{array}{l}0,058 \\
0,045 \\
0,039\end{array}$ & $\begin{array}{l}6 / 24 \\
4 / 20 \\
4 / 19\end{array}$ & $\begin{array}{l}0,085 \\
0,105 \\
0,096\end{array}$ & $\begin{array}{l}1,47 \\
2,33 \\
2,46\end{array}$ \\
\hline \multirow[t]{2}{*}{$\begin{array}{l}\text { Broncho- } \\
\text { pneunomie } \\
\text { (Maus) }\end{array}$} & 1 & $\begin{array}{l}\text { i. } n . \\
\text { i. } n . \\
\text { i. } n .\end{array}$ & $\begin{array}{r}1000 \\
10000 \\
100000\end{array}$ & $\begin{array}{l}10 . \\
10 . \\
10 .\end{array}$ & $\begin{array}{l}3 / 7 \\
1 / 8 \\
6 / 7\end{array}$ & $\begin{array}{l}0,130 \\
0,153 \\
0,093\end{array}$ & $\begin{array}{l}0,37 \\
0,81 \\
0,44\end{array}$ & $\begin{array}{l}1 / 8 \\
1 / 8 \\
0 / 8\end{array}$ & $\begin{array}{l}0,356 \\
0,188 \\
0,211\end{array}$ & $\begin{array}{l}1 / 8 \\
0 / 8 \\
3 / 8\end{array}$ & $\begin{array}{l}0,270 \\
0,298 \\
0,1 \cup 4\end{array}$ & $\begin{array}{l}0,76 \\
1,59 \\
0,49\end{array}$ \\
\hline & 2 & i. $n$. & 1000 & 10. & $10 / 22$ & 0,110 & 0,61 & $3 / 23$ & 0,180 & - & - & - \\
\hline \multirow[t]{2}{*}{$\begin{array}{l}\text { Lympho- } \\
\text { granuloma } \\
\text { inguinale }\end{array}$} & 1 & $\begin{array}{l}\text { i. c. } \\
\text { i. c. } \\
\text { i. c. }\end{array}$ & $\begin{array}{r}50 \\
100 \\
500\end{array}$ & $\begin{array}{l}10 . \\
10 . \\
10 .\end{array}$ & $\begin{array}{l}3 / 8 \\
3 / 7 \\
2 / 7\end{array}$ & $\begin{array}{l}0,130 \\
0,116 \\
0,127\end{array}$ & $\begin{array}{l}0,88 \\
0,70 \\
0,64\end{array}$ & $\begin{array}{l}0 / 6 \\
1 / 8 \\
0 / 6\end{array}$ & $\begin{array}{l}0,147 \\
0,166 \\
0,2 \cup 0\end{array}$ & $\begin{array}{l}0 / 5 \\
1 / 6 \\
1 / 7\end{array}$ & $\begin{array}{l}0,176 \\
0,173 \\
0,153\end{array}$ & $\begin{array}{l}1,20 \\
1,04 \\
0,77\end{array}$ \\
\hline & 2 & $\begin{array}{l}\text { i. c. } \\
\text { i. c. } \\
\text { i. c. }\end{array}$ & $\begin{array}{r}50 \\
100 \\
500\end{array}$ & $\begin{array}{l}10 . \\
10 . \\
10 .\end{array}$ & $\begin{array}{l}3 / 15 \\
3 / 16 \\
8 / 15\end{array}$ & 0,125 & $\begin{array}{l}0,79 \\
0,94 \\
0,69\end{array}$ & $\begin{array}{l}2 / 13 \\
2 / 15 \\
4 / 14\end{array}$ & & $\begin{array}{l}0 / 13 \\
0 / 14 \\
1 / 12\end{array}$ & $\begin{array}{l}0,299 \\
0,367 \\
0,183\end{array}$ & $\begin{array}{l}1,89 \\
2,64 \\
1,33\end{array}$ \\
\hline \multirow[t]{3}{*}{ Ektromelie } & 1 & i. n. & 1000 & 15. & $15 / 24$ & 0,075 & 1,12 & $12 / 22$ & 0,067 & - & - & - \\
\hline & 2 & i. $n$. & 100 & 15. & $11 / 18$ & 0,071 & 0,50 & $6 / 20$ & 0,141 & - & - & - \\
\hline & 3 & i. pl. & 100 & 15. & $10 / 24$ & 0,060 & 0,55 & $3 / 24$ & 0,11 & - & - & - \\
\hline \multirow[t]{2}{*}{ Influenza } & 1 & $\begin{array}{l}\text { i. } n . \\
\text { i. n. } \\
\text { i. } n .\end{array}$ & $\begin{array}{r}100 \\
1000 \\
10000\end{array}$ & $\begin{array}{l}13 . \\
13 . \\
13 .\end{array}$ & $\begin{array}{l}2 / 4 \\
2 / 4 \\
4 / 4\end{array}$ & $\begin{array}{l}0,083 \\
0,080 \\
0,045\end{array}$ & $\begin{array}{l}0,45 \\
0,65 \\
0,43\end{array}$ & $\begin{array}{l}0 / 4 \\
0 / 4 \\
0 / 4\end{array}$ & $\begin{array}{l}0,185 \\
0,123 \\
0,105\end{array}$ & $\begin{array}{l}0 / 4 \\
0 / 4 \\
1 / 4\end{array}$ & $\begin{array}{l}0,175 \\
0,163 \\
0,088\end{array}$ & $\begin{array}{l}0,95 \\
1,33 \\
0,84\end{array}$ \\
\hline & 2 & $\begin{array}{l}\text { i. } n . \\
\text { i. } n . \\
\text { i. } n .\end{array}$ & $\begin{array}{r}100 \\
1000 \\
10000\end{array}$ & $\begin{array}{l}13 . \\
13 . \\
13 .\end{array}$ & $\begin{array}{l}2 / 6 \\
0 / 7 \\
1 / 8\end{array}$ & $\begin{array}{l}0,083 \\
0,067 \\
0,10\end{array}$ & $\begin{array}{l}0,25 \\
0,30 \\
0,45\end{array}$ & $\begin{array}{l}0 / 6 \\
1 / 8 \\
0 / 7\end{array}$ & $\begin{array}{l}0,332 \\
0,223 \\
0,220\end{array}$ & $\begin{array}{l}0 / 8 \\
0 / 8 \\
0 / 8\end{array}$ & \begin{tabular}{l|}
0,29 \\
0,231 \\
0,186
\end{tabular} & $\begin{array}{l}0,87 \\
1,04 \\
0,85\end{array}$ \\
\hline \multirow[t]{2}{*}{$\begin{array}{l}\text { Lymphocy- } \\
\text { täreChorio- } \\
\text { meningitis }\end{array}$} & 1 & $\begin{array}{l}\text { i. c. } \\
\text { i. c. } \\
\text { i. c. }\end{array}$ & $\begin{array}{r}100 \\
1000 \\
10000\end{array}$ & $\begin{array}{l}14 . \\
14 . \\
14 .\end{array}$ & $\begin{array}{c}10 / 10 \\
9 / 9 \\
9 / 11\end{array}$ & $\begin{array}{l}0,0 \\
0,0 \\
0,017\end{array}$ & $\begin{array}{l}0,0 \\
0,0 \\
0,52\end{array}$ & $\begin{array}{l}4 / 12 \\
0 / 12 \\
8 / 12\end{array}$ & $\begin{array}{l}0,091 \\
0,119 \\
0,033\end{array}$ & $\begin{array}{l}1 / 12 \\
0 / 12 \\
2 / 7\end{array}$ & $\begin{array}{l}0,133 \\
0,138 \\
0,094\end{array}$ & $\begin{array}{l}1,46 \\
1,16 \\
2,85\end{array}$ \\
\hline & 2 & i. c. & 100 & 21. & $10 / 18$ & 0,048 & 0,49 & 8,18 & 0,097 & - & - & - \\
\hline \multirow[t]{2}{*}{ Louping ill } & 1 & $\begin{array}{l}\text { i. c. } \\
\text { i. c. }\end{array}$ & $\begin{array}{r}1000 \\
10000\end{array}$ & $\begin{array}{l}21 . \\
21\end{array}$ & $\begin{array}{l}2 / 8 \\
4 / 8\end{array}$ & $\begin{array}{l}0,074 \\
0,044\end{array}$ & $\begin{array}{l}0,50 \\
0,32\end{array}$ & $\begin{array}{l}0 / 7 \\
0 / 8\end{array}$ & $\begin{array}{l}0,149 \\
0,136\end{array}$ & $\begin{array}{l}0 / 6 \\
0 / 6\end{array}$ & $\begin{array}{l}0,167 \\
0,130\end{array}$ & $\begin{array}{l}1,12 \\
0,96\end{array}$ \\
\hline & 2 & i. c. & 1000 & 14. & $17 / 24$ & 0,038 & 0,33 & $2 / 24$ & 0,115 & - & - & - \\
\hline
\end{tabular}

Tab.1. Zusammenstellung der Versuchsergebnisse.

zwischen Serum, Periston (Kollidon) und verschiedenen Farbstoffen, ferner über die Ausscheidung von an Periston gebundenen Farbstoffen ergeben haben. Er hat weiter auf die von Schubert ${ }^{6}$ festgestellte entgiftende Wirkung des Kollidons gegenüber verschiedenen Bakterientoxinen aufmerksam gemacht. Ein solcher antitoxischer Effekt ist bei den Virusarten wohl aus-

${ }^{6}$ R. S c hubert, Dtsch. med. Wschr. 1948, 551.

${ }^{7}$ E. Gildemeister u. E. Ha a gen, Dtsch. med. Wschr. 1940 I, 878. [1944]. zuschalten, denn Toxinbildung wurde bisher nur bei den Rickettsien (Rickettsia prowazeki ${ }^{7}$ ) und bläschenförmigen Virusarten (Lymphogranuloma inguinale-, Meningopneumonitis- und Mäusebronchopneumonie-Virus ${ }^{8}$ ) festgestellt. Bei diesen Erregern ist aber die Virulenzabschwächung in unseren Versuchen keineswegs besonders ausgesprochen. Daß es sich bei der Virulenzabschwächung verschiedener Virusarten in vitro durch Kollidon um eine spezifische Wirkung handelt, ist wohl nicht anzunehmen. Bisher fehlt noch eine Erklärung für dieses Phänomen. Vielleicht liegt 
Über die Kapselvirus-Krankheit (S.338).

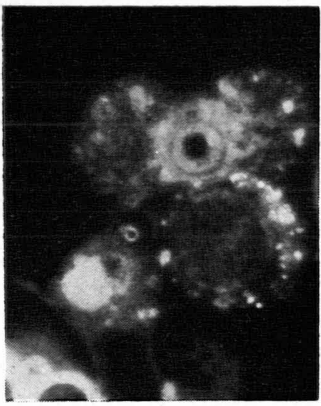

Abb. 1.

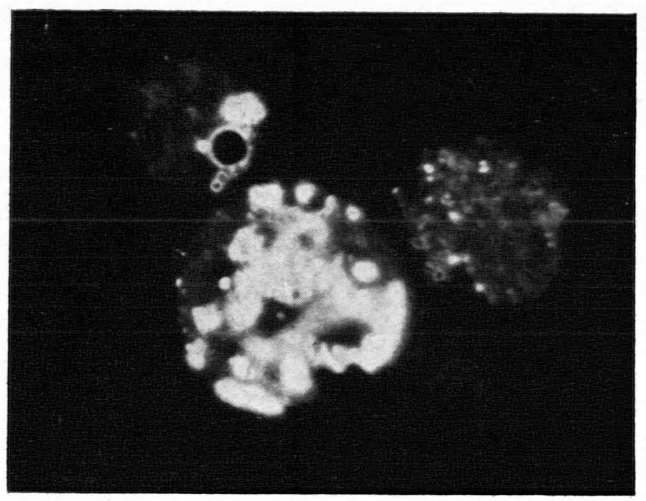

Abb. 2 .

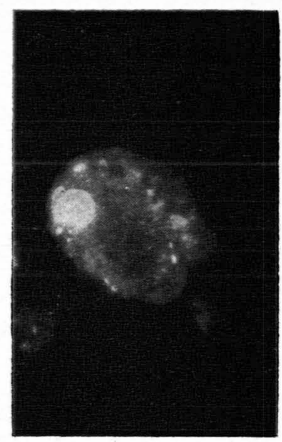

Abb. 3.
Abb. 1-5. Blutzellen von Cacoecia murinana. In den erkrankten Zellen sieht man leuchtend weiße, verschieden große blasenförmige Gebilde, die dicht mit Viruskapseln gefüllt sind. Ortholux Mikroskop 1/12 a, Dunkelfeld Ölkondensor 1,20. Vergr. etwa 1000/1.

Abb. 6*. Viruskapseln von Cacoecia murinana.

* Die Abb. 6-13 sind elektronenoptisch etwa 9000-fach nachvergrößert auf $25000 / 1$. $50 \mathrm{KV}$ elektrostatisch, Negativbilder.

Abb. 7. Cacoecia murinana. Viruskapseln, aus welchen durch alkalische Behandlung die Kapselvirusstäbchen herausgeschlüpft sind, wodurch stäbchenförmige Löcher bzw. Hohlräume in den Viruskapseln entstehen.

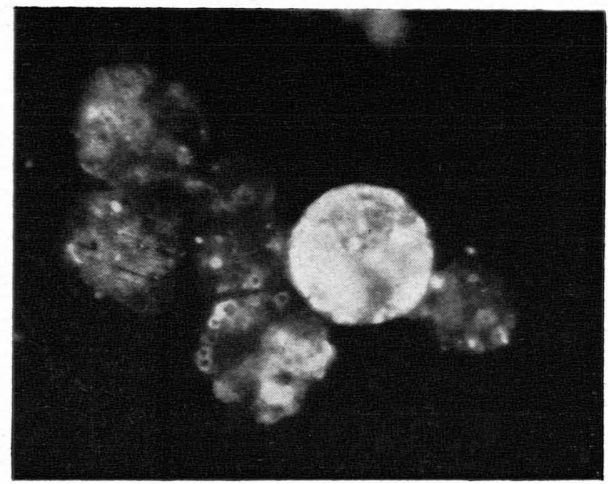

Abb. 4.

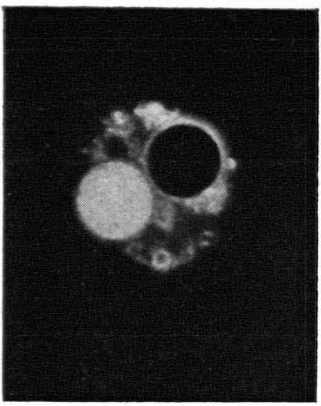

Abb. 5 .

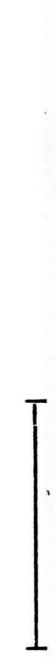

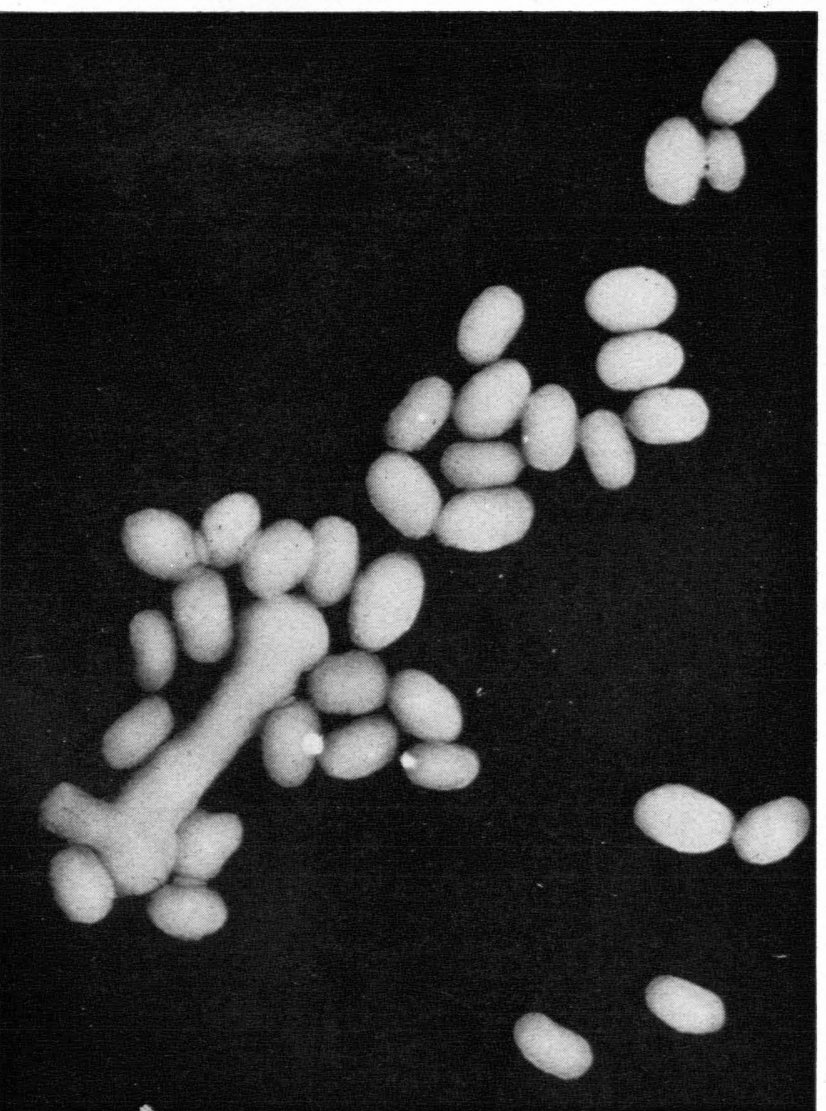

Abb. 6.

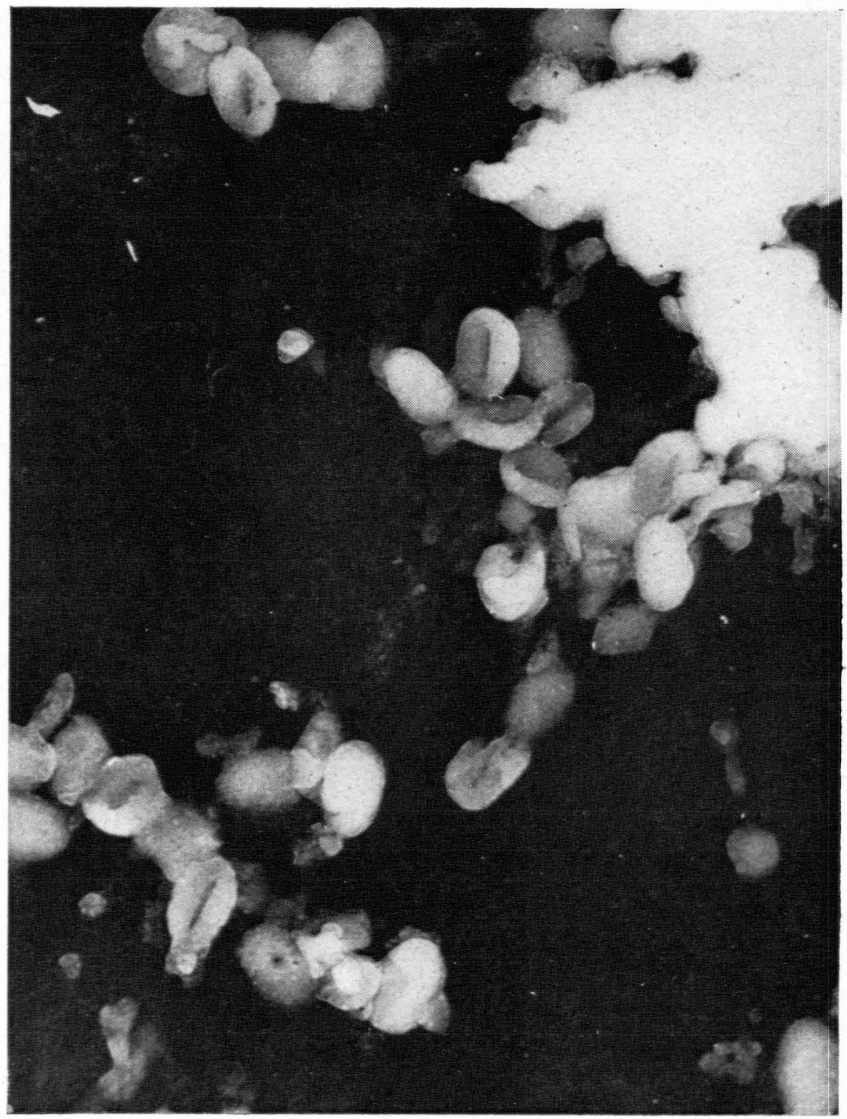

Abb. 7. 

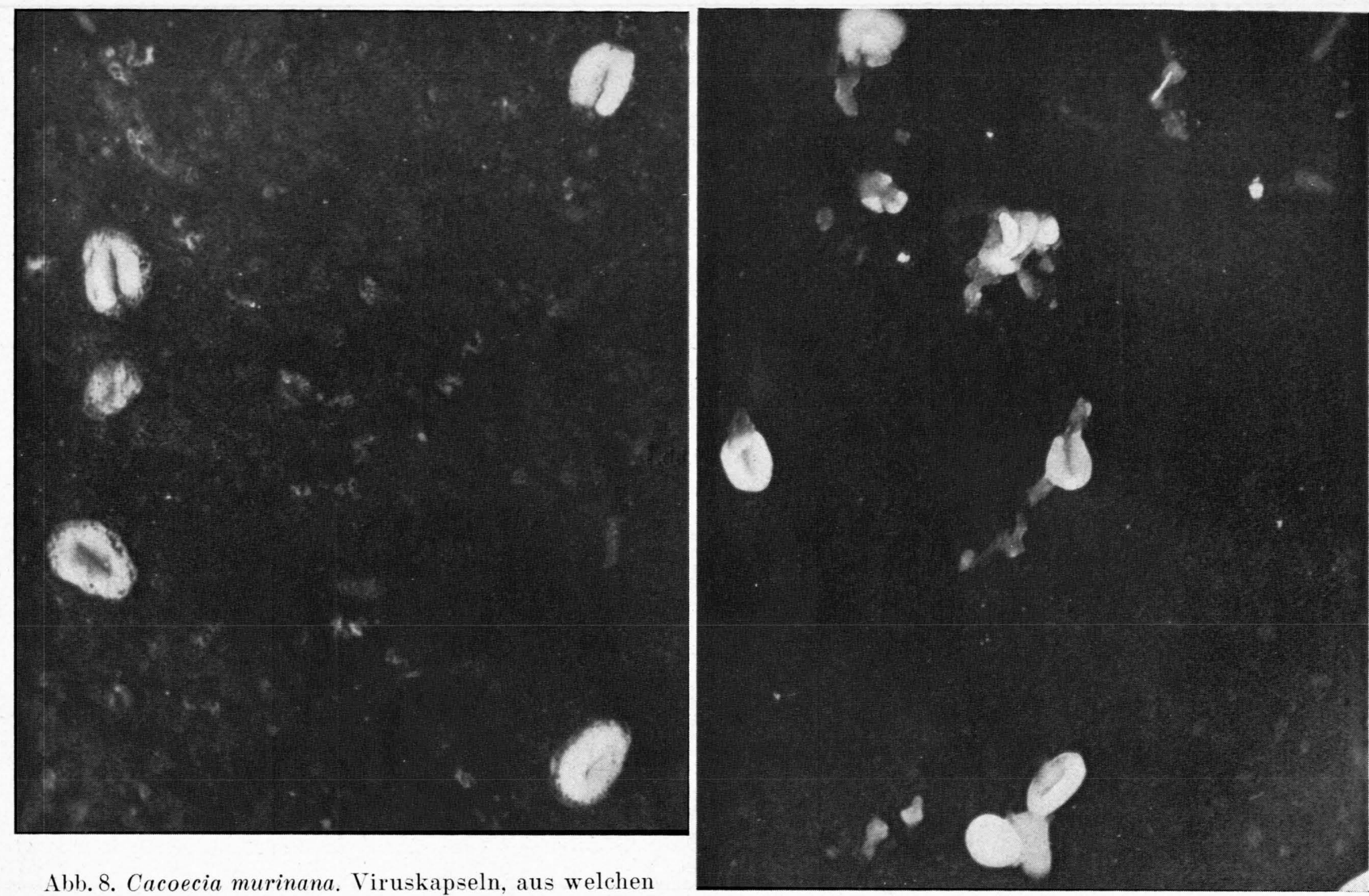

Abb. 8. Cacoecia murinana. Viruskapseln, aus welchen durch alkalische Behandlung die Kapselvirusstäbchen herausgeschlüpft sind, wodurch stäbchenförmige Löcher

bzw. Hohlräume in den Viruskapseln entstehen.

Abb. 9. Cacoecia murinana. Viruskapseln, aus denen Virusstäbchen schlüpfen. Rechts oben einzelne freie Virusstäbchen.
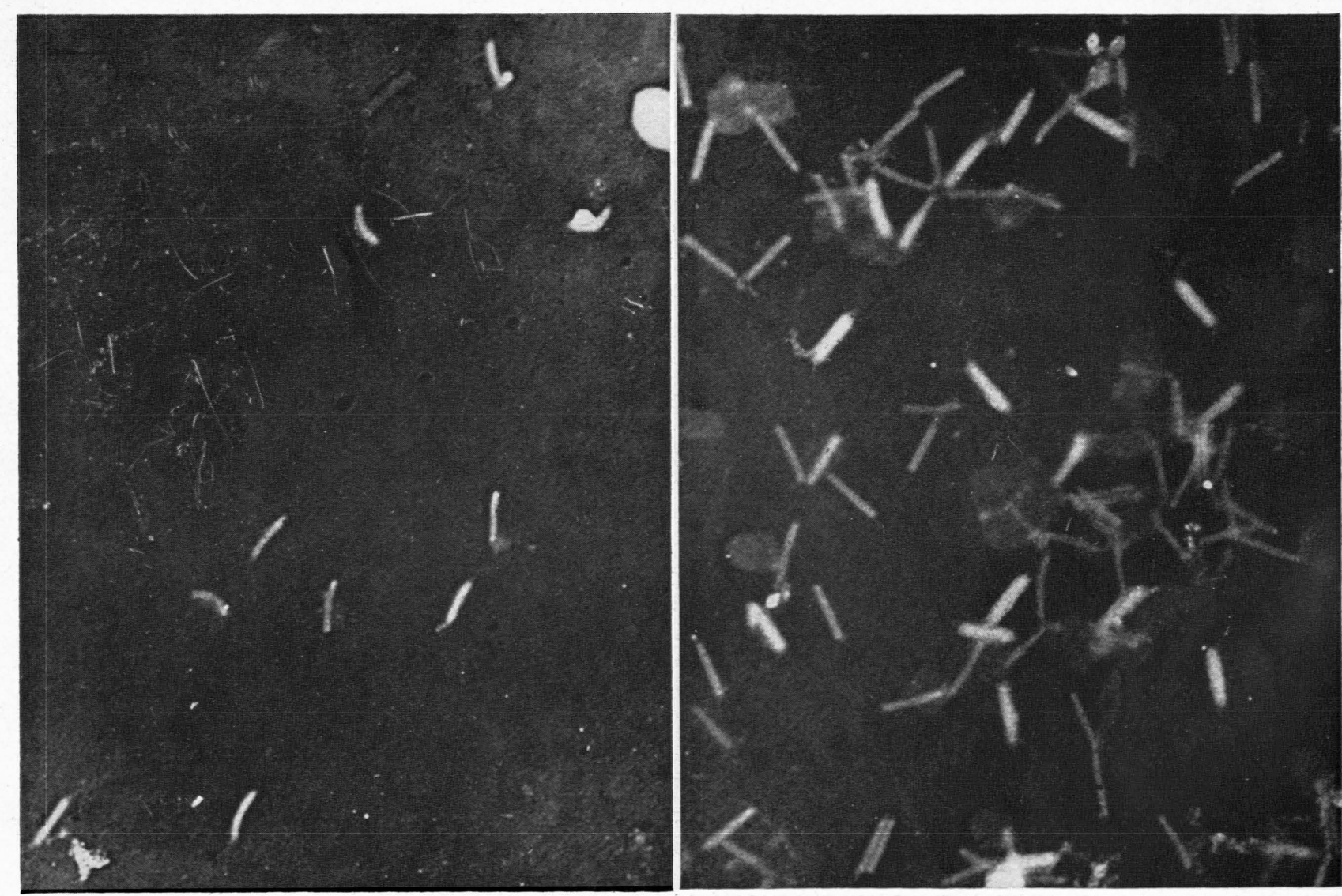

Abb. 10. Isolierte Kapselvirusstäbchen mit Querstrukturen.

Abb. 11. Bombyx mori, Polyedervirus. Einzel- und Doppelstäbchen mit Querstrukturen. 

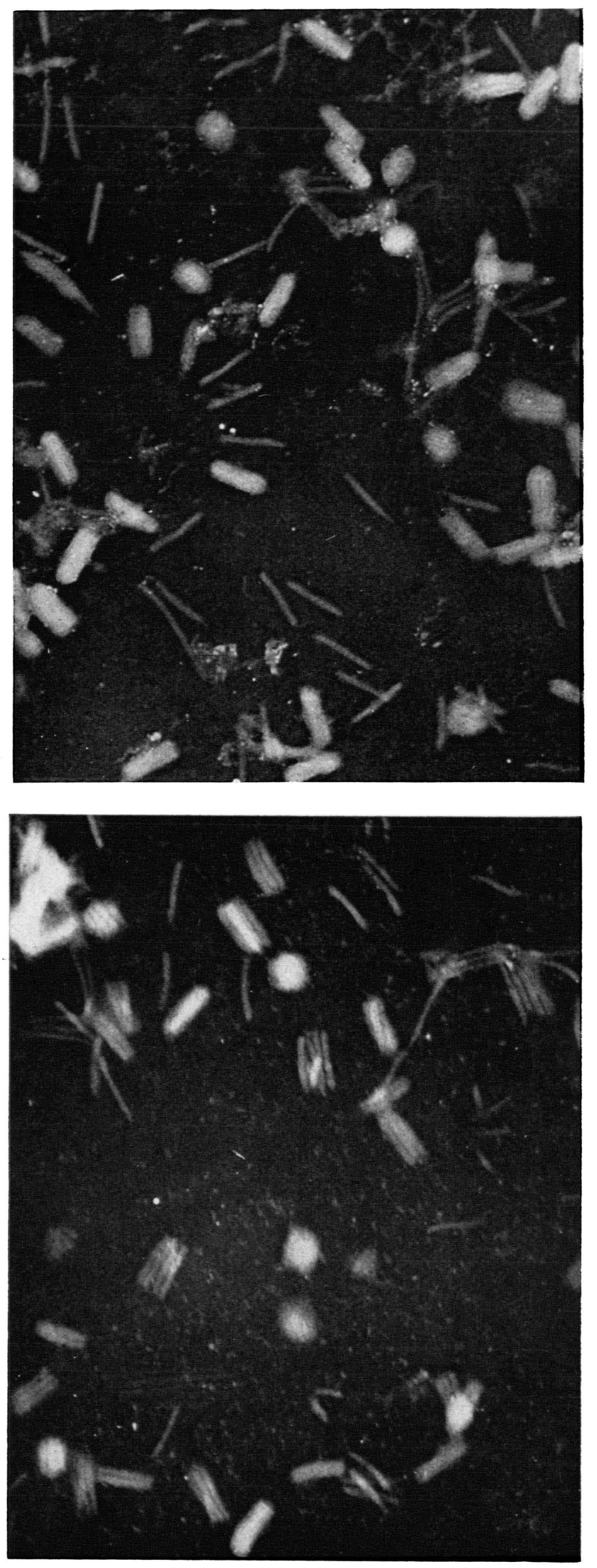

Abb. 12-13. Porthetria dispar, Polyedervirus. Einzelne und gebündelte Stäbchen. 


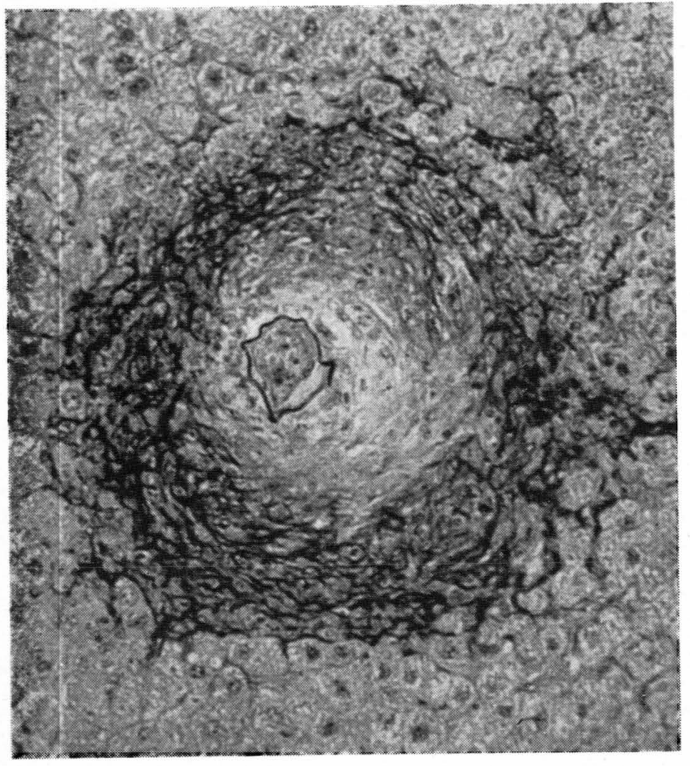

Abb. 1. Leber, Maus, Bilharzia mansoni-Infektion. Junger Pseudotuberkel im Querschnitt, ein Ei mit lebendem Miracidium enthaltend. - Susa, Van Gieson, Vergr. 200/1.



Abb. 2. Leber, Maus, B. mansoni-Infektion. Alter hyalinisierter Pseudotuberkel im Längsschnitt, Eischale von pigmenthaltiger Riesenzelle phagorevtiert. - Suca. Van Fieson. Vergr. 200/1.

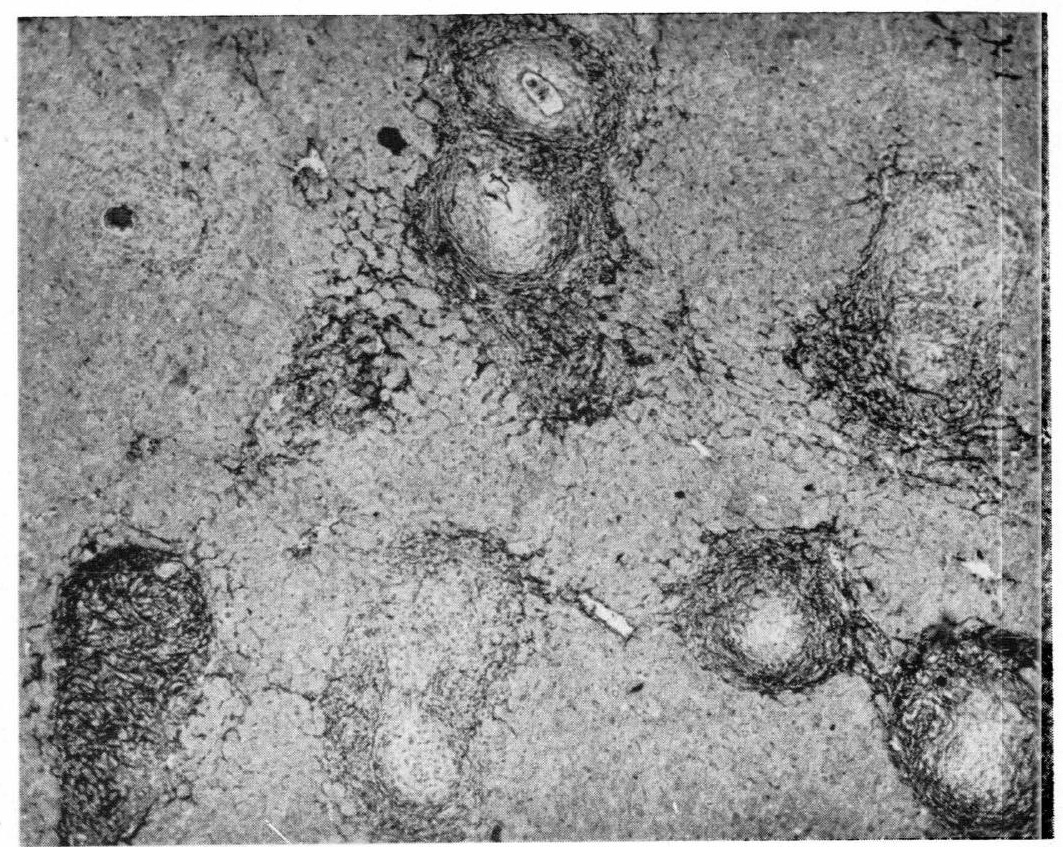

Abb. 3. Leber, Maus, B. mansoni-Infektion. Frischere Pseudotuberkel mit kollateraler Sklerose. - Susa, Azan, Vergr. 70/1.
Abb. 4. Leber, Maus, B. mansoni-Infektion. Kleinfleckige, scharf begrenzt Narhen. 7 Monate narh Miracilbehandlung. - Susa. Azan, Vergr. 70/1. 
eine entsprechende chemische Beeinflussung vor, wie sie von Bovet, Jacob, Ducrot und Courvoisie $r^{\theta}$ in dem Kollidonspaltprodukt $\boldsymbol{x}$-Pyrrolidon zur Erklärung der antitraumatischen Wirkung des Kollidons angenommen wird. B o vet, Courvoisier und D u crot ${ }^{10}$ konnten den Nachweis erbringen, daß Ratten, welche mit Kollidon vorbehandelt waren, traumatische Einflüsse überlebten, an denen die Kontrollen zugrunde gingen.

Es wäre auch zu erwägen, ob es sich bei der Kollidonwirkung in vitro nicht um eine rein physikalische Umhüllung des Virus handelt. In diesem Falle ließe sich die Virulenzabschwächung so erklären, daß die Elementarkörperchen infolge der Kollidonschutzhülle nicht alle zu gleicher Zeit infizieren, da sie erst nach Freiwerden infektiös werden. Dies würde eine protrahierte Infektion mit kleinen Dosen über einen größeren Zeitraum bedeuten. Auf den gleichen Mechanismus wird auch die Wirkungsweise des Fleckfieberimpfstoffes nach Laigret und Durand ${ }^{11}$ zurückgeführt. Bei diesem Impfstoff handelt es sich um virulente Rickettsien in getrocknetem, pulverisiertem Gehirn (Ratte oder Meerschweinchen), die mit Eigelb und öl umhüllt sind.

${ }^{2}$ M. D. B ovet, R. J a c ob, R. Ducrot u. S. C o u r v o i s i e r, C. R. hebd. Séances Acad. Sci. 224, 496 [1947].

10 M. D. Bovet, S. Courvoisier u. M. R. D u c r o t, C. R. hebd. Séances Acad. Sci. 224, 70 [1947].

${ }_{11} \mathrm{~J} . \mathrm{L}$ a i g r e t u. R. D u r a nd, Arch. Inst. Pasteur Algérie 25, 82 [1936].

\section{Zur Pathologie der Leber bei experimenteller Schistosomiasis mansoni der Maus}

\section{Von Rudolf Gönnert und Hans Werner Altmann}

Aus dem Chemotherapeutischen Institut der Farbenfabriken Bayer, Leiter: Prof. Dr. W. Kikuth, und dem Pathologischen Institut der Universität Freiburg i. Br., Direktor: Prof. Dr. F. Bü chner

(Z. Naturforschg. 3 b. 345-348 [1948]; eingegangen •am 17. November 1948)

Die vorläufige Mitteilung bringt einen kurzen Überblick über die Pathogenese der Leberveränderungen bei der experimentellen Bilharzia-mansoni-Infektion der Maus. Die pathologischen Bilder sind z. Tl. durch die Wurmtoxine und das Bilharzia-Pigment - ein Hämoglobin-Abbauprodukt aus dem Darme der Bilharzien -, vor allem jedoch durch die mit dem Blutstrom in die Leber verschleppten Wurmeier bedingt. Um diese bilden sich typische Pseudotuberkel, deren verschiedene Entwicklungsstadien kurz beschrieben werden. Bei älteren Infektionen kann es, je nach Verteilung des reaktiven Bindegewebes, zu verschiedenen Formen-der Lebercirrhose kommen. Die Analyse der Leberveränderungen wurde besonders durch die Einbeziehung der Stadien nach Ausheilung der Infektion mit dem neuen Heilmittel Miracil ermöglicht. Hierbei ergaben sich eingehende Untersuchungen über das Schicksal der durch das Medikament abgetöteten Würmer. Wird die Behandlung bei nicht zu alter Infektion vorgenommen, so kann es bei der Maus zu einer fast vollständigen Restitution der Leber im Verlauf über eines Jăhres kommen. Hierbei treten für die spezielle Pathologie der Leberzellen sehr charakteristische Veränderungen an Kern und Plasma auf.

$\mathrm{U}$ nter Schistosomiasis versteht man eine medizinisch sehr wichtige parasitäre Erkrankung des Menschen, welche in bestimmten tropischen und subtropischen Gebieten endemisch auftritt und durch gewisse Arten der zu den Saugwürmern gehörenden Gattung Bilharzia (Schistosoma) hervorgerufen wird. Die Parasiten leben, je nach Art, in verschiedenen Abschnitten der Abdominal. venen und rufen, ihrem Sitz entsprechend, jeweils wohl charakterisierte Krankheitserscheinungen hervor (Darm- oder Blasen-Schistosomiasis). Für unsere Untersuchungen stand der Erreger der Darm-Schistosomiasis, Bilharzia mansoni Sambon 1907, zur Verfügung, der experimentell auf die weiße Maus übertragen werden kann. Er macht, wie alle diese Trematoden, einen komplizierten Entwicklungsgang durch, der mit einem Wirtswechsel (Endwirt-Schnecke-Endwirt) ein- 